\title{
An intelligent approach to take care of mother and baby health
}

\author{
Mohammad Nasser Uddin, Mohammad Jahangir Alam, Md. Nurul Mustafa \\ Department of Computer Science and Information Technology, Southern University Bangladesh, Bangladesh
}

\begin{tabular}{l} 
Article Info \\
\hline Article history: \\
Received Nov 1, 2017 \\
Revised Aug 3, 2018 \\
Accepted Aug 30, 2018 \\
\hline Keywords: \\
Expectant mother \\
Health sector \\
Mother and baby care \\
Smart phone \\
User interface
\end{tabular}

\begin{abstract}
This is the era of technology and is widely used in every sector. In Bangladesh the use of technology is increasing day by day in many sectors. Health sector is one of them. This research is designed and developed to help our pregnant mother to get weekly information on development and conditions of their health and the growing child inside their womb. This system will notify expectant mothers automatically about their health checkup date and time. It provides general and special health information to the expectant mothers. It is designed with user friendly interface so that an expectant mother can use this system very effectively. This system allows a unique secure login system and provides a unique suggestion to the expectant mothers. This system is very user friendly and useful.
\end{abstract}

Copyright $\odot 2019$ Institute of Advanced Engineering and Science. All rights reserved.

Corresponding Author:

Mohammad Nasser Uddin,

Department of Computer Science and Information Technology,

Southern University Bangladesh,

739/A Mehidibag Road, Chittagong, Bangladesh.

Email: nasseruddin83@gmail.com

\section{INTRODUCTION}

A healthy baby can give immense happiness to a family. So before being a mother, she should plan many things that will ensure a healthy pregnancy and a healthy baby. This research is made for the preparation of pregnant women. The goal of the system is to help the expectant mothers for healthy pregnancy and increase their self-care awareness. Most of the expectant mothers need to visit their doctor regularly for a check-up and keep track of their health care. However, the doctor might not be able to always keep track on them and give them immediate feedback. Thus, this application is to encourage the expectant mothers to keep track on their health anywhere and anytime they wish. In addition, this application will also provide some guidance to nutrition and some workout for the pregnant women. Hence, this may help them save their time, improve their healthcare and satisfaction and reduce costs.

NasimKhozouie, FaranakFotouhi-Ghazvini, BehrouzMinaei-Bidgoli published a paper with the title "Ontological MobiHealth System" in the journal of Indonesian Journal of Electrical Engineering and Computer Science [1]. In this research, they introduced a context-aware mobile application for patient monitoring at home. This framework has three steps. First, they collected physiological, activity, and environmental data using WBAN. In the next step, they extracted context situations from raw data and created a dynamic ontology with OWL language. In this research, context situations include activity, blood pressure, body temperature, ratio of oxygen in blood $(\mathrm{SpO} 2)$ and ECG features, which are extracted from normal sinus rhythm for a human heart on ECG (e.g., HR, P, Q, R, S, P-R, QRS, S-T, and QT). In the third step, they programmed rules and alarm management using java code for android operating systems (smart phones). Finally, this framework was evaluated in three scenarios.

Rohini R. Rao, Krishna moorthiMakkithaya published a paper with the title "Learning from a Class Imbalanced Public Health Dataset: a Cost-based Comparison of Classifier Performance" on the journal of International Journal of Electrical and Computer Engineering (IJECE) [2]. In this Paper they described that 
this paper work is relevant to public health policy makers who can use the classifiers to predict the occurrence of chronic disease in the population and also identify the factors that are correlated with chronic diseases. The classifiers will help health care providers in improving their prognosis, diagnosis and treatment plans.

Jong Won Lee, Han Kil Kim, Hoe Kyung Jung published a paper with the title "User Analysis Mechanisms based Mobile Fitness System" in the journal of International Journal of Electrical and Computer Engineering (IJECE) [3]. In this paper they showed that the proposed system is available to recommend the appropriate data to the user by analyzing the information of user body. Analysis of user application mechanism is based on three recommendation algorithm. The first recommendation algorithm analyzes the user's body information, and likes to calculate the exercise BMI index. The second recommendation algorithm analyzes the BMR value and metabolic activity of the user like metabolic rate and tells quantity of exercise. The third recommendation algorithm that tells the trainers belonging to the same BMI group, such as the user, and makes recommendations to other users using exercise equipment that fits their BMI index. This user may be provided with the data for strength training. Then the data makes it possible to perform the efficient exercise for the user.

Sherin Sebastian, Neethu Rachel Jacob, YeduManmadhan, Anand V. R., M. J. Jayashree, published a paper with the title "Remote Patient Monitoring System" in the journal of International Journal of Distributed and Parallel Systems (IJDPS) [4]. In this research they described the android application which is developed using UI easy to understand and check the health status of the patient. Emergency alarm, Alerts, Remainders, Notification alarms help the doctor to take timely decision at emergency situation.

Mackillop, Loerup, Bartlett, Gibson, Kenworthy, published a paper with the title "Development of a real-time smartphone solution for the management of women with or at high risk of gestational diabetes" in the journal of Journal of Diabetes Science and Technology [5]. In this paper they developed an intuitive, interactive, reliable, and accurate management system to record BG measurements and deliver management of GDM remotely.

\section{OBJECTIVES OF THE PROPOSED SYSTEM}

The paper describes an intelligent approach to take care of mother and baby health which has the following objectives:

a. To calculate current week of pregnancy.

b. To get information about baby.

c. To track pregnancy week by week.

d. To track weight and baby kicks.

e. To calculate baby born due date.

f. To make notes with pregnancy symptoms

g. To track instant health condition.

h. To take doctor's appointment.

i. To get emergency service i.e. ambulance call, telemedicine.

\section{TECHNOLOGY AND TOOLS ARE USED IN THIS RESEARCH}

\subsection{Tools and techniques}

Smart phone: A smartphone is a mobile phone that includes advanced functionality beyond making phone calls and sending text messages. Most smartphones have the capability of displaying photos, playing videos, check and sending e-mail, and surf the Web. Modern smartphones, such as the iPhone and Android based phones can run third-party applications, which provide limitless functionality [6].

Android: Android app is a software application running on the Android platform. As the Android platform is built for mobile devices, a typical Android app is designed for a smartphone or a tablet PC running on the Android OS [7].

Web server: A Web server is a system that delivers content or services to the end users over the Internet. A Web server consists of a physical server, server operating system (OS) and software used to facilitate HTTP communication. A Web server is also known as an Internet server [8].

Database: A database is a structured collection of data. It may be anything from a simple shopping list to a picture gallery or the vast amounts of information in a corporate network. To add, access, and process data stored in a computer database, we need a database management system such as MySQL Server. Since computers are very good at handling large amounts of data, database management systems play a central role in computing, as standalone utilities, or as parts of other applications [9]. 


\subsection{Algorithm}

Quick sort: Quicksort is a fast sorting algorithm, which is used not only for educational purposes, but widely applied in practice. On the average, it has no complexity making quicksort suitable for sorting big data volumes. The idea of the algorithm is quite simple and once we realize it, we can write quicksort as fast as bubble sort [10]. In this project Quick sort algorithm has been used to find doctor's suggestion using user's symptoms from the symptoms database.

The divide-and-conquer strategy is used in quicksort. Below the recursion step is described:

1. Choose a pivot value. We take the value of the middle element as pivot value, but it can be any value, which is in range of sorted values, even if it doesn't present in the array.

2. Partition. Rearrange elements in such a way that all elements which are lesser than the pivot go to the left part of the array and all elements greater than the pivot, go to the right part of the array. Values equal to the pivot can stay in any part of the array. Notice that array may be divided in non-equal parts.

3. Sort both parts. Apply quicksort algorithm recursively to the left and the right parts.

Example: Sort $\{1,12,5,26,7,14,3,7,2\}$ using quicksort.

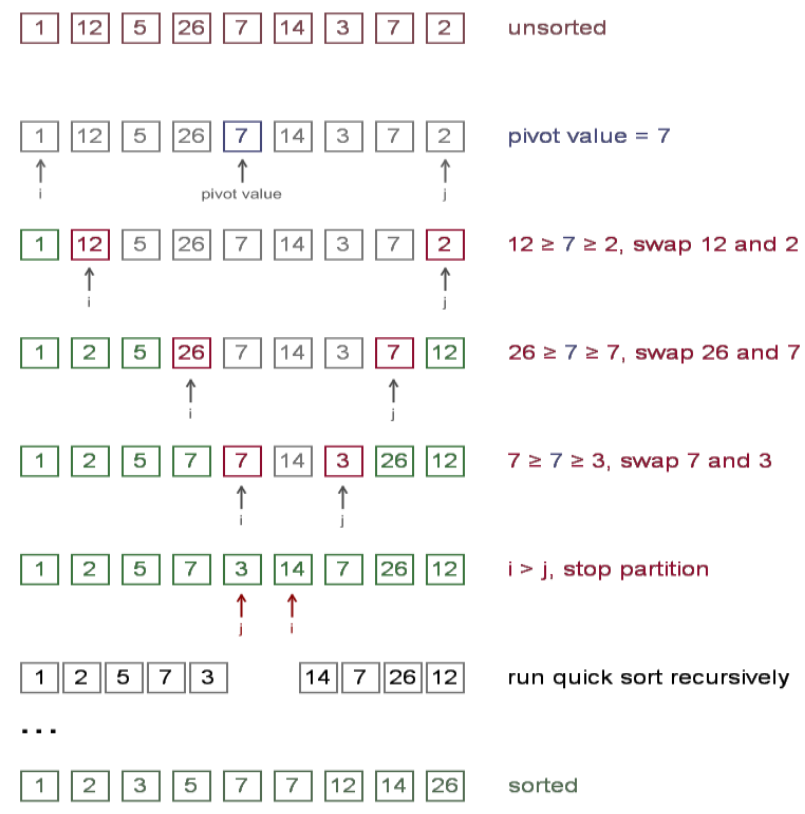

\section{SYSTEM ARCHITECTURE}

In the following proposed system architecture there are two diagrams, one is concept diagram and the other one is block diagram. The conceptual diagram shown in Figure 1.

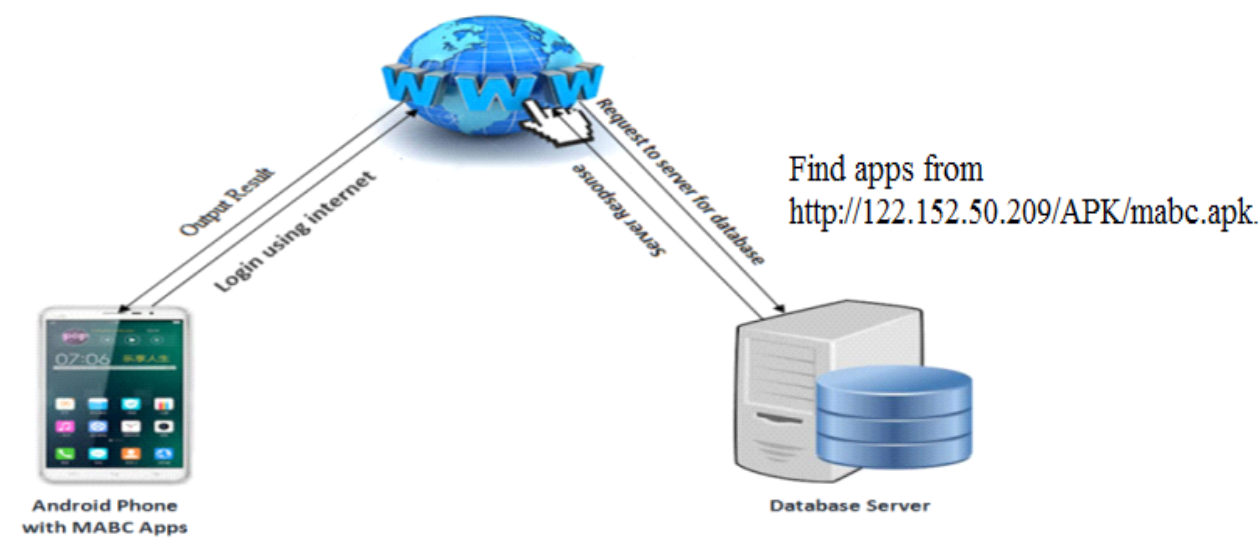

Figure 1. Conceptual diagram of an intelligent approach to take care of mother and baby health 


\section{IMPLEMENTATION}

This project has two applications, one application is for Android phone and the other application is web application API for http connection with database. If someone wants to get supportive information and services, he or she should download the apps and install into their mobile phones. After completing the installation, the user should create an account by registration and then login to the system.

\subsection{Flowchart}

Flowchart for an intelligent approach to take care of mother and baby health shown in Figure 2 .

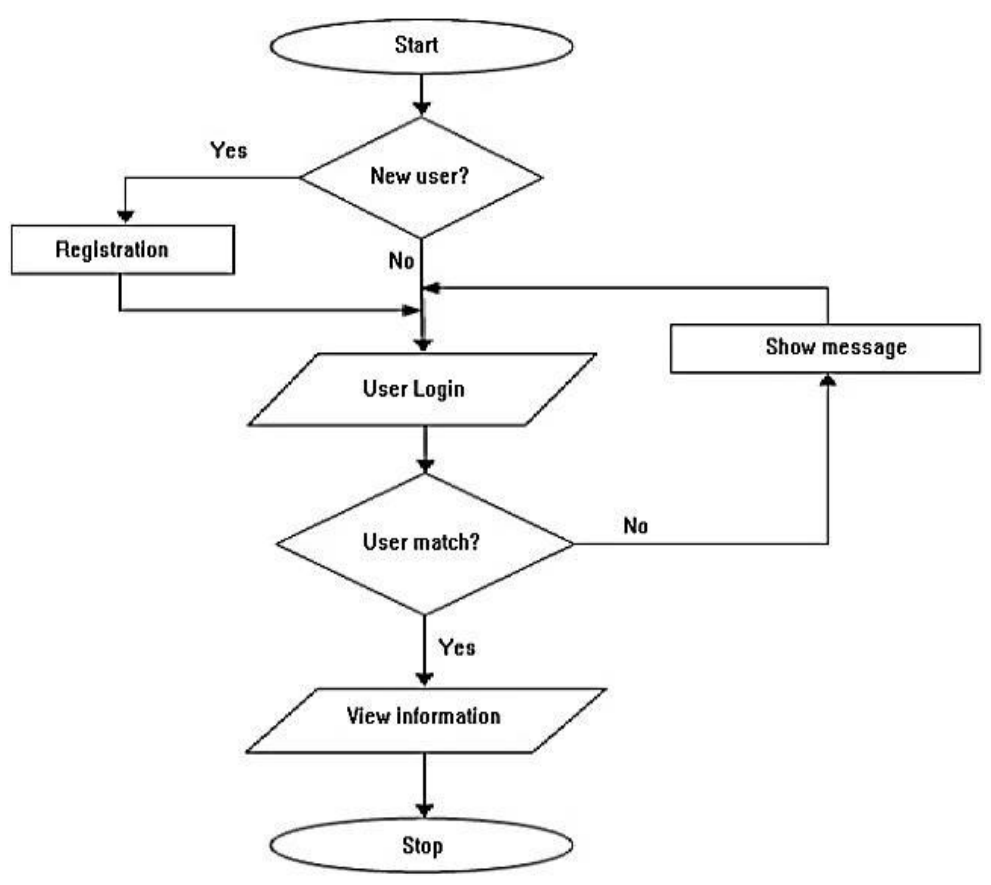

Figure 2. Flowchart for an intelligent approach to take care of mother and baby health

The flow chart shows the total procedure of an intelligent approach to take care of mother and baby health system. A user should follow some steps to get support and make decision:

a. Start

b. If new user then

1. Registration

c. User login

d. Check match found

e. If match found then

1. Show my self condition

2. Show my baby condition

3. Show symptoms and suggestion

4. Show other information

f. If match not found then

1. Show error message

g. Stop

\subsection{Interface of the system}

User interface is the front-end application view to which user interacts in order to use the software. User can manipulate and control the software as well as hardware. This is the front page of mobile application before registration as Figure 3. The user should register this application by clicking the Registration button. After clicking the registration page of the mobile application, the user has to type full name, e-mail address, phone number, and last menstruation date into the text box and type password into the 
password text box as like as Figure 4. After entering information and password, click register button, then this application will be registered.

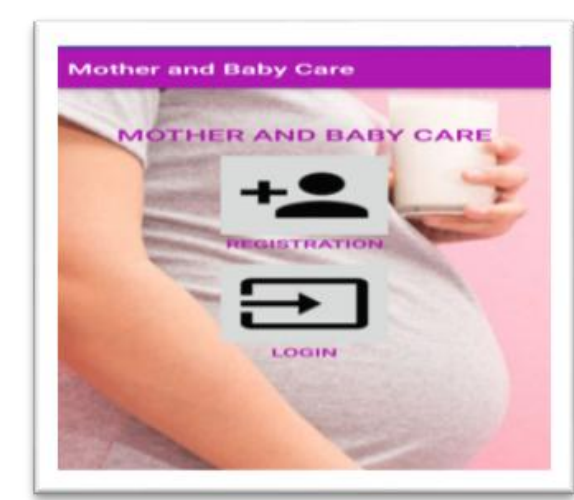

igure 3. User interface for an intelligent approach to take care of mother and baby health

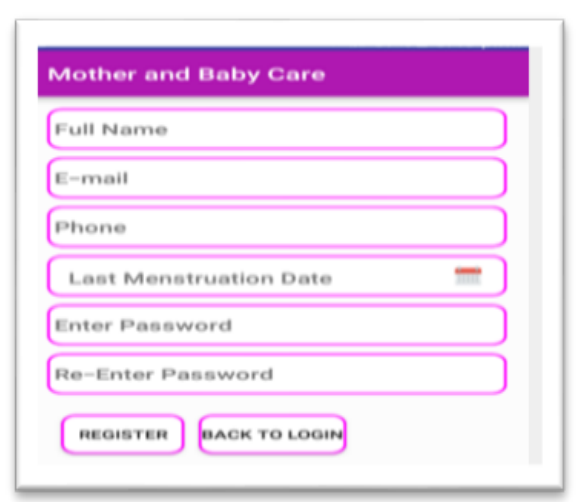

Figure 4. Registration form

\section{RESULT AND ANALYSIS}

To access to the application, the user has to login into the system by entering e-mail address, phone number and password as Figure 5. After successful login a user will get relevant information and be able to access to services and tools as Figure 6. Among the menus when a user clicks on myself button it will show the pregnant mother's current week health condition as liks as Figure 7. If the mother wants to know about her baby, she has to tap on mybaby menu and then she will get her baby's current week health conditions inside the mother's womb as on Figure 8. A mother can also calculate the probable baby born due date using the baby born date calculator by giving mother's last menstruation date as Figure 9.

A mother can track what to do in the current month such as when to go health checkup, when to take vaccine and dosages, other important activities by the calendar tools. The selected dates indicate what to do in the particular day as Figure 10. The Figure 11 shows all probable symptoms list during her pregnancy period. The intelligences are included in this section. It is more interactive and dynamic. A mother may get suggestion by selecting symptom of own feelings and physical condition automatically. After selecting symptoms click on "click for doctor tips" for getting suggestions as Figure 12. These suggestions are prepared by sorting (Quick sort algorithms) symptoms according to diseases from the database. By selecting the symptoms, they can seek out the percentage according to selection of the highest number of symptoms under a disease. Then a quick sort is performed among the percentage and found the largest percentage of the symptoms selection of a diseases. Then the system shows the possible diseases list according to percentage of symptoms selection and display the related suggestion from the related database.

The user or mother can appoint a doctor or call ambulances in critical situation using this app just tapping on call or message button easily. Figure 13 shows how to appoint the doctor and display text message after successful sending request.

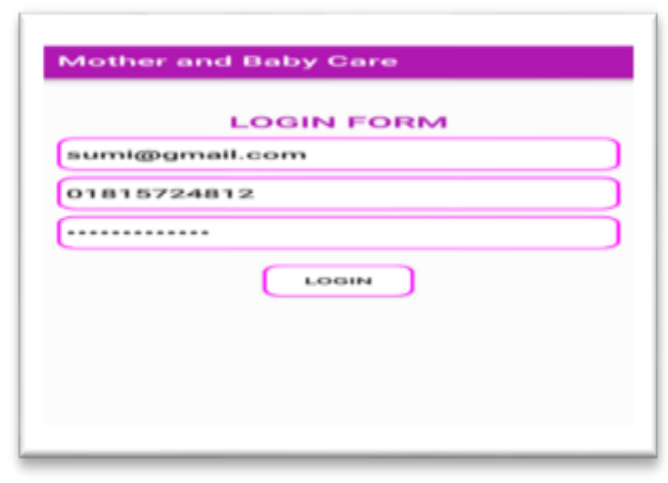

Figure 5. Login page with user Id and password

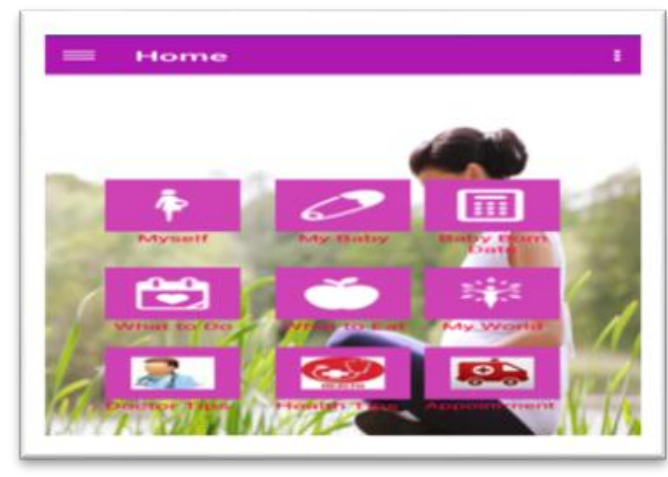

Figure 6. Menu 


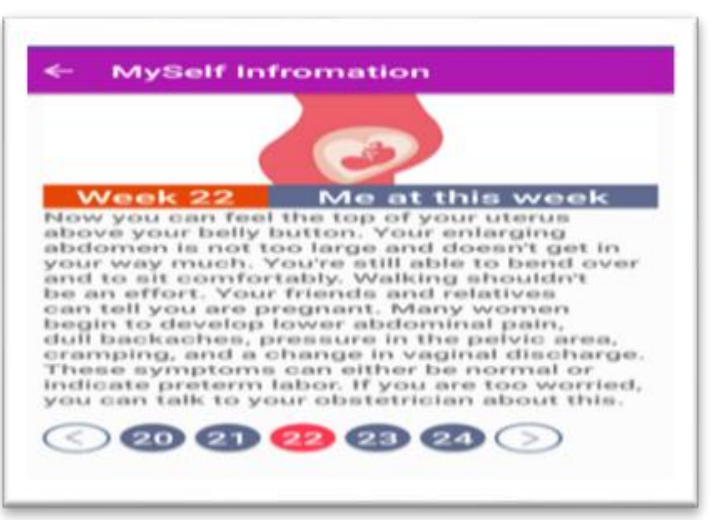

Figure 7. My self information page

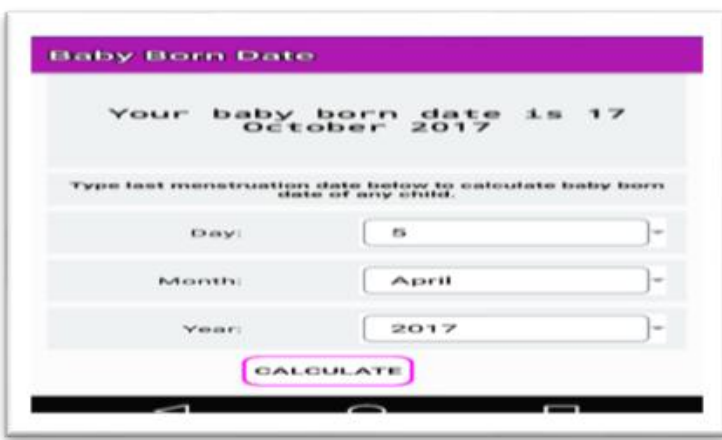

Figure 9. Baby born due date calculator

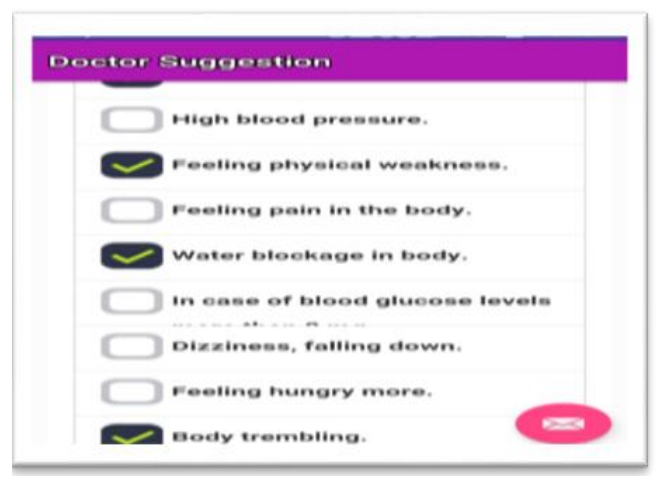

Figure 11. Symptoms selection

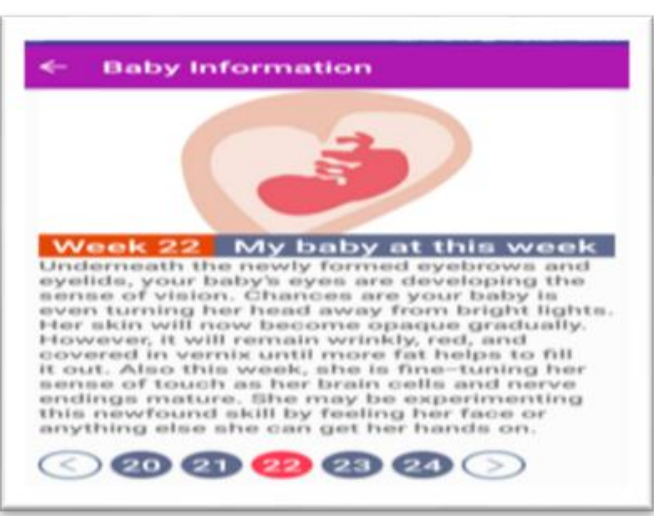

Figure 8. My baby information page

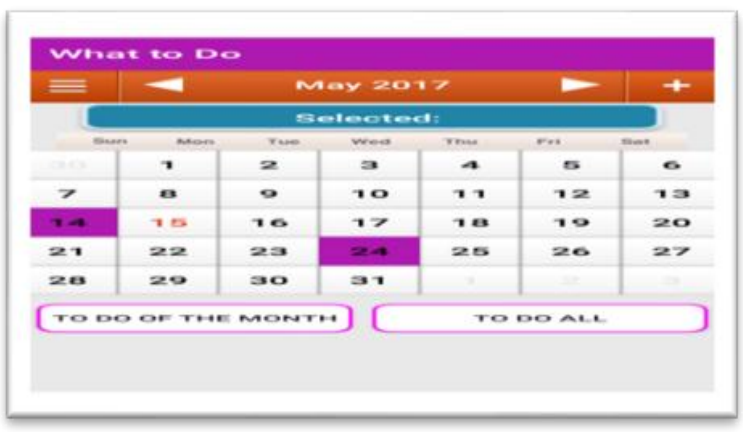

Figure 10. To do page

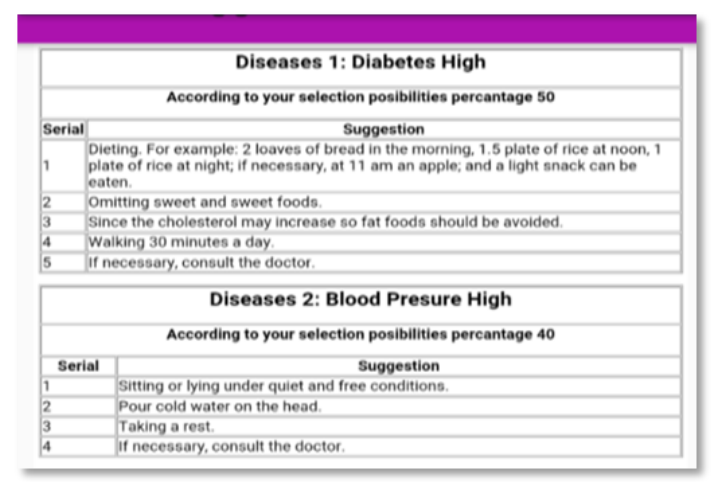

Figure 12. Doctor suggestions page

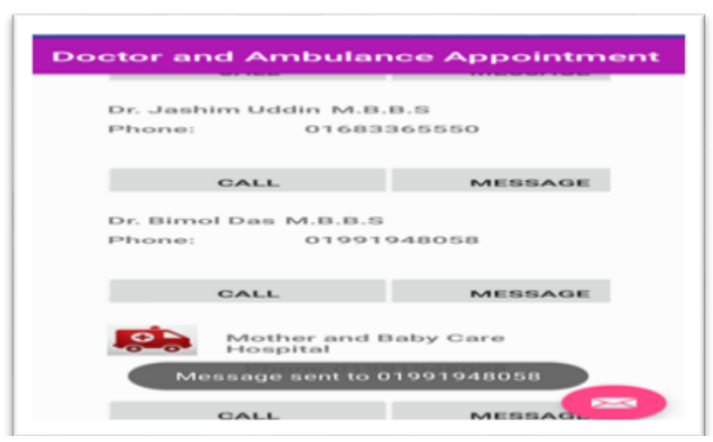

Figure 13. Doctor appointment by sending message 


\section{COMPARISON}

The comparisons with other systems shown in Table 1.

Table 1. The Comparisons with Other Systems

\begin{tabular}{|c|c|c|c|c|}
\hline System name & Security & Interactive & Notification & Instant Service \\
\hline Pregnancy Tracker & Medium & Yes & No & No \\
\hline Pregnancy Guide & Low & No & No & No \\
\hline An intelligent approach to take care of mother and baby health & High & Yes & Yes & Yes \\
\hline
\end{tabular}

\section{CONCLUSION}

In this research, we have developed an intelligent approach to take care of mother and baby health. We have conducted this research to help our pregnant mother raise their self-care awareness. Using this application, an expectant mother can be worried free due to its many useful features. This system will work as an assistant as well as a guide to make tension free of the expectant mother. It is very nice and the user can easily use this application. Any people can track expectant mother health condition and get solution any time using this application. We have experimented in different Android phones and it has proved perfect. We think users will be benefited by using this application.

\section{REFERENCES}

[1] NasimKhozouie, FaranakFotouhi-Ghazvini, BehrouzMinaei-Bidgoli, "Ontological MobiHealth System,” Indonesian Journal of Electrical Engineering and Computer Science(IJEECS) Vol. 10, No. 1, pp. 309 319, April 2018.

[2] Rohini R. Rao, KrishnamoorthiMakkithaya, "Learning from a Class Imbalanced Public Health Dataset: a Cost-based Comparison of Classifier Performance," International Journal of Electrical and Computer Engineering (IJECE) Vol. 7, No. 4, pp. 2215 2222, August 2017.

[3] Jong Won Lee, Han Kil Kim, Hoe Kyung Jung, "User Analysis Mechanisms based Mobile Fitness System," International Journal of Electrical and Computer Engineering (IJECE) Vol. 6, No. 6, pp. 3154 3160, December 2016.

[4] Sherin Sebastian, Neethu Rachel Jacob, YeduManmadhan, Anand V. R., M. J. Jayashree, "Remote Patient Monitoring System," International Journal of Distributed and Parallel Systems (IJDPS), Vol.3, No.5, pp.99-110, September 2012.

[5] Mackillop, Loerup, Bartlett, Gibson, Kenworthy, "Development of a real-time smartphone solution for the management of women with or at high risk of gestational diabetes," Journal of Diabetes Science and Technology, Vol. 8(6), pp. 1105-1114, July 2014.

[6] Internet:https://techterms.com/definition/smartphone,July 30, 2010 [July 05, 2017].

[7] Internet: https://www.techopedia.com/definition/25099/android-app [July 07, 2017].

[8] Internet: https://www.techopedia.com/definition/4928/web-server [July 07, 2017].

[9] Internet:https://dev.mysql.com/doc/refman/5.7/en/what-is-mysql.html, August 15, 2016 [June 21, 2017].

[10] Internet:http://www.geeksforgeeks.org/quick-sort, January 07, 2014 [June 25, 2017].

\section{BIOGRAPHIES OF AUTHORS}

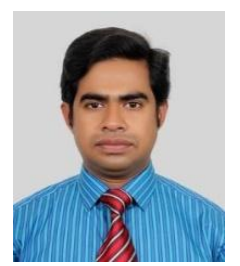

Mohammad Nasser Uddin was born on 02 February1983 at Chittagong, Bangladesh. He has completed his M.Sc.( Physics) in 2004 under the National University of Gazipur, Dhaka, Bangladesh and post-graduation (MSc in CSIT) in 2017 from Southern University Bangladesh, Chittagong. He is a Lecturer of Computer Science at Chittagong Port Women College, Chittagong under Chittagong Port Authority. He is also an MCSA certified.

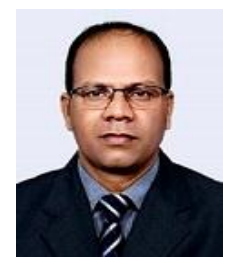

Mohammad Jahangir Alam was born in 1978 in Chittagong. He has completed post graduation (MSc in CSIT) in 2008 from Southern University Bangladesh, Chittagong. He is an Assistant Professor at the department of Computer Science and Information Technology in Southern University Bangladesh, Chittagong. He has published more than 10 technical papers in reputed national, International Journals and Conferences. 


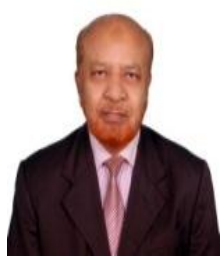

Dr. Md.Nurul Mustafa was born on 6th June 1948 at Cox's Bazar, Bangladesh. He has completed his M.Sc.( Physics) in 1970 from the University of Chittagong, Bangladesh. He joined as a Faculty Member at the same Department in 1973. He obtained his higher degree from Concordia University, Montreal, Canada in 1986 and came back and became Professor in 1992. He shifted to Computer Science and Engineering Department as Professor and founder Chairman in 2001 at the same University. After retirement from the University of Chittagong in 2014, Dr. Mustafa has been serving as a Professor of Computer Science at Southern University Bangladesh. He published more than forty-five research articles in the field of Radiation Physics and Computer Science in National and International Journals. 